\title{
Medical insurance claims associated with international business travel
}

\author{
Bernhard Liese, Kenneth A Mundt, Linda D Dell, Lorraine Nagy, Bernard Demure
}

\begin{abstract}
Objectives-Preliminary investigations of whether 10884 staff and consultants of the World Bank experience disease due to work related travel. Medical insurance claims filed by 4738 travellers during 1993 were compared with claims of nontravellers.

Methods-Specific diagnoses obtained from claims were analysed overall (one or more $v$ no missions) and by frequency of international mission $(1,2-3$, or $\geqslant 4)$. Standardised rate of claims ratios (SRRs) for each diagnostic category were obtained by dividing the age adjusted rate of claims for travellers by the age adjusted rate of claims for non-travellers, and were calculated for men and women travellers separately.
\end{abstract}

Results-Overall, rates of insurance claims were $80 \%$ higher for men and $18 \%$ higher for women travellers than their non-travelling counterparts. Several associations with frequency of travel were found. SRRs for infectious disease were $1.28,1.54$, and 1.97 among men who had completed one, two or three, and four or more missions, and 1.16, 1.28, and 1.61, respectively, among women. The greatest excess related to travel was found for psychological disorders. For men SRRs were $2.11,3.13$, and 3.06 and for women, SRRs were $1.47,1.96$, and 2.59 .

Conclusions-International business travel may pose health risks beyond exposure to infectious diseases. Business travellers file medical claims at a greater rate than non-travellers, and for many categories of disease, the rate of claims increases with frequency of travel. The reasons for higher rates of claims among travellers are not well understood. Additional research on psychosocial factors, health practices, time zones crossed, and temporal relation between travel and onset of disease is planned.

\section{(Occup Environ Med 1997;54:499-503)}

Keywords: travel; epidemiology; medical insurance claims

Correspondence to:

Dr Bernhard Liese, Director, Health Services Department,

The World Bank, 700 18th

Street, Room MC2-429,

Washington, DC 20433.

Accepted 23 January 1997

Sudden changes in climate, environment, and time zones, and the related physical and psychological stress associated with international business travel may pose health risks beyond exposure to endemic diseases. The medical literature identifies various diseases which have been associated with travel. The most common of these are the infectious and parasitic diseases, ${ }^{1}$ including sexually transmitted diseases, ${ }^{23}$ and intestinal ${ }^{4}$ and dermatological conditions. ${ }^{5-7}$ Non-communicable conditions have also been reported, such as thromboses and embolisms, ${ }^{89}$ psychiatric disorders, ${ }^{10-12}$ and injuries. ${ }^{13}$ Relatively little, however, is known about the effects of frequent international business travel on the general health of employees of a large international institution.

The World Bank sends its staff, based mostly in Washington DC, on 18000 international business missions each year. In 1993, 4738 operational staff of the World Bank consisting of regular employees, and long term consultants, logged nearly 250000 person-days on missions, usually to destinations in Asia, Africa, and Latin America. To investigate whether staff of the World Bank experienced more disease due to work related travel, we compared the prevalence of a wide range of diagnoses from medical insurance claims filed on behalf of travellers with those of nontravellers, and by frequency of international missions.

\section{Materials and methods}

STUDY DESIGN

A cross sectional study based on records was conducted on all 10884 insured staff of the World Bank in 1993. Expense reports generated by staff after each mission were used $(a)$ to identify travellers and $(b)$ to assign them to categories of frequency of travel (one, two to three, and four or more missions). Age and sex were found from personnel records.

The independent insurance company, which manages the Bank's medical insurance plan, provided claims data in aggregate for diagnostic categories, stratified by sex, age category ( $<35,35$ to 44,45 to 54 , and 55 years or older), and by frequency of travel. Diagnoses were coded according to the ninth revision of the international classification of diseases (ICD-9). No more than one claim per person was included for each three digit ICD-9 code.

\section{STATISTICAL ANALYSIS}

Standardised rate of claims ratios (SRRs) and $95 \%$ confidence intervals ( $95 \% \mathrm{CIs}$ ) were calculated for men and women travellers separately. Age adjusted rates of claims for both 
Table 1 Age distribution of male and female travellers and non-travellers at the World Bank in 1993

\begin{tabular}{lllll}
\hline Characteristic & Men (\%) & Women (\%) & Total & Men: Women \\
\hline Missions (n): & & & & \\
0 & $2324(41.0)$ & $3822(73.3)$ & $6146(56.5)$ & $0.6: 1$ \\
1 & $608(10.7)$ & $592(11.4)$ & $1200(11.0)$ & $1.0: 1$ \\
$2-3$ & $991(17.5)$ & $433(8.3)$ & $1424(13.1)$ & $2.3: 1$ \\
₹4 & $1749(30.8)$ & $365(7.0)$ & $2114(19.4)$ & $4.8: 1$ \\
$\quad$ Total staff & $5672(100.0)$ & $5212(100.0)$ & $10884(100.0)$ & $1.1: 1$ \\
Age: & & & & \\
$\quad$ Non-travellers: & & & & \\
$\quad$ <55 & $745(32.1)$ & $1243(32.5)$ & $1988(32.3)$ & $0.6: 1$ \\
$\quad 35-44$ & $742(31.9)$ & $1245(32.6)$ & $1987(32.3)$ & $0.6: 1$ \\
$\quad 45-54$ & $563(24.2)$ & $1017(26.6)$ & $1580(25.7)$ & $0.6: 1$ \\
$\quad>54$ & $274(11.8)$ & $317(8.3)$ & $591(9.6)$ & $0.9: 1$ \\
Travellers: & & & & \\
$\quad<35$ & $460(13.7)$ & $378(27.2)$ & $838(17.6)$ & $1.2: 1$ \\
$\quad 35-44$ & $885(26.4)$ & $555(39.9)$ & $1440(30.4)$ & $1.6: 1$ \\
$\quad 45-54$ & $1349(40.3)$ & $390(28.1)$ & $1739(36.7)$ & $2.4: 1$ \\
$\quad>54$ & $654(19.5)$ & $67(4.8)$ & $721(15.2)$ & $9.8: 1$ \\
\hline
\end{tabular}

travellers (overall and by frequency of mission) and non-travellers were calculated by summing age specific rates of claims weighted according to the age distribution of all staff of the World Bank. The SRR for each diagnostic category was obtained by dividing the age adjusted rate of claims for travellers (overall and by frequency of mission) by the corresponding age adjusted rate of claims for non-travellers. Ninety five per cent confidence intervals (95\% CI) were estimated and reported for all SRRs.

\section{Results}

Three times as many men than women travelled on missions. Women, who comprise nearly half of all employees at the Bank, represented $29 \%$ of travellers and $62 \%$ of nontravellers in 1993. Among the 1390 female travellers, $43 \%$ completed only one mission in 1993 compared with $18 \%$ of the 3348 male travellers. Although the number of female travellers decreased with increasing number of missions, the number of male travellers increased with the number of missions travelled (table 1).

As a group, travellers $(n=4738)$ were older than non-travellers $(n=6146)$ (table 1$)$. Because of these differences by age and sex, all subsequent results were controlled for age by stratification and are presented separately for men and women.

Among both men and women, the distribution of claims across categories of diagnoses was similar for travellers and non-travellers. However, travellers filed claims at a higher rate than non-travellers: the overall rate of claims was $80 \%$ higher among male travellers and $18 \%$ higher among female travellers. Among male travellers, rates of claims were increased for all major categories of diagnoses examined (table 2). Among female travellers (table 3), the relative excesses were $<25 \%$ for all major

Table 2 Frequency of selected claims diagnoses and standardised claims rate ratios (SRRs) (95\% CIs) overall and according to frequency of travel for male travellers

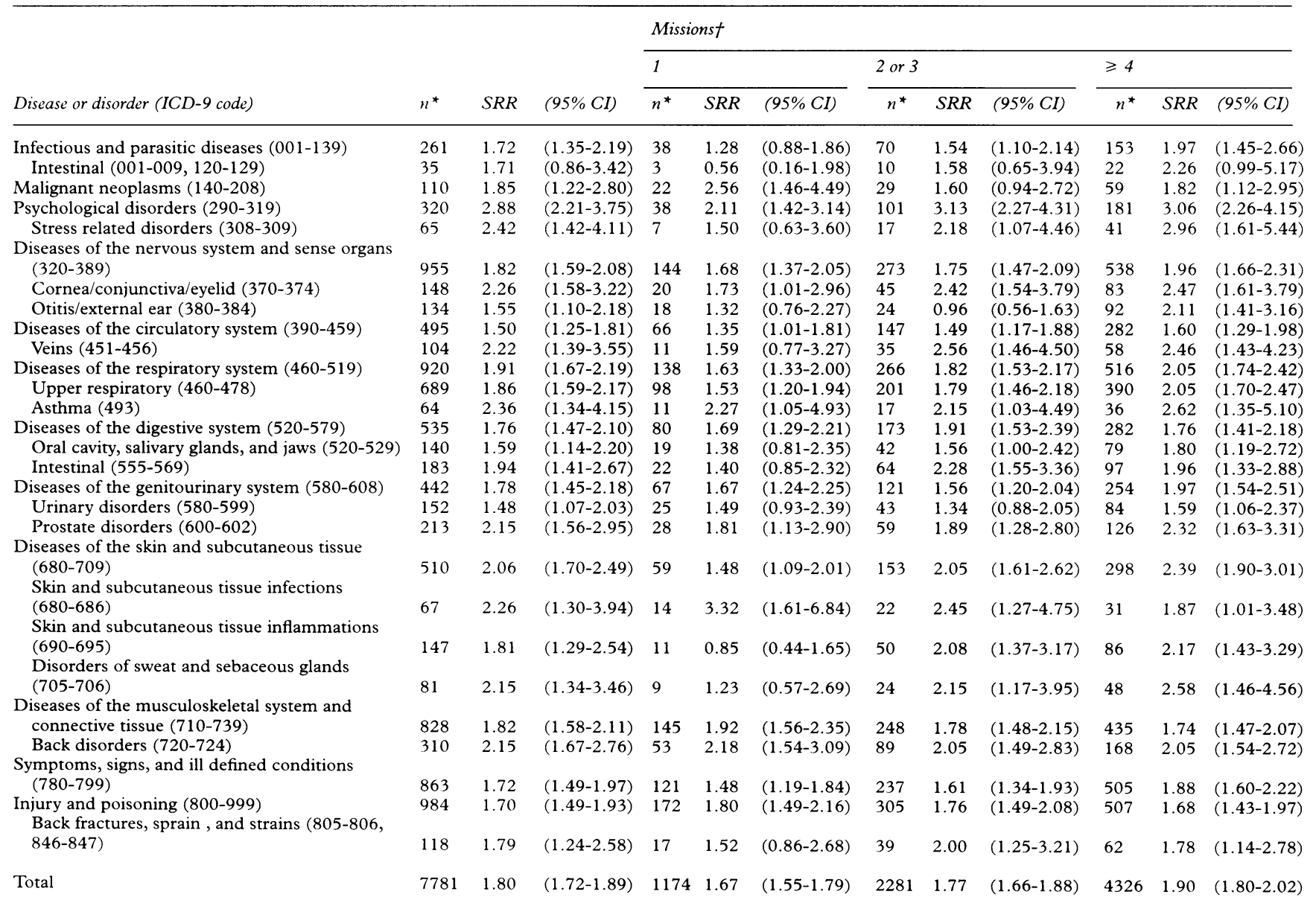

* Number of claims filed by travellers.

† Reference category is non-travellers. 
Table 3 Frequency of selected claims diagnoses and standardised claims rate ratios (SRRs) (95\% CIs) overall and according to frequency of travel for female travellers

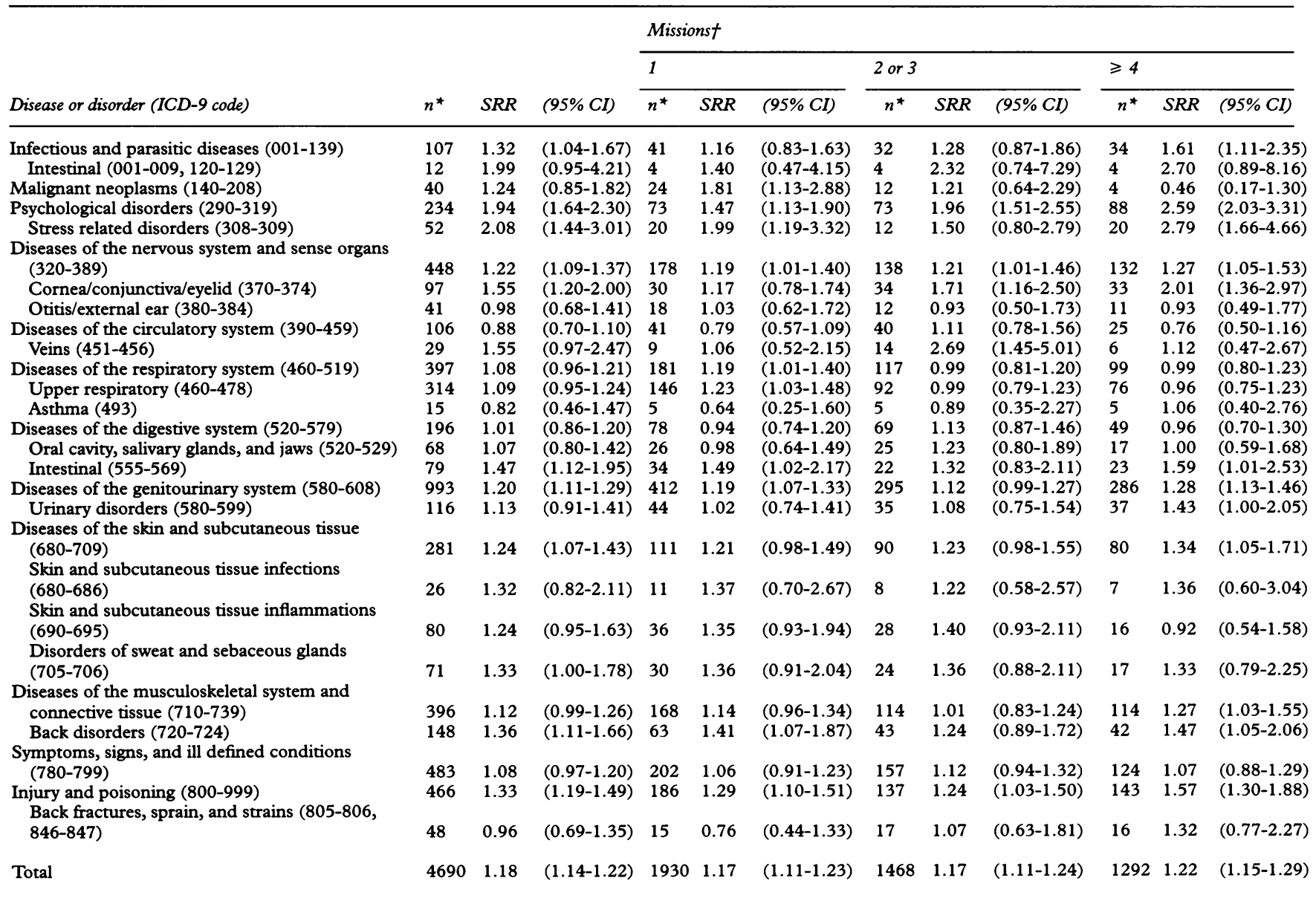

^ Number of claims filed by travellers.

† Reference category is non-travellers.

disease categories except psychological disorders $\quad(S R R=1.94)$, infectious diseases $(S R R=1.32)$, and injuries $(S R R=1.33)$.

EVALUATION OF TRENDS IN MEDICAL CLAIMS WITH INCREASING TRAVEL

Not all excesses were correlated with frequency of mission, and several diagnostic categories, such as diseases of the circulatory system, upper respiratory tract, and oral cavity, showed a relation for men but not women travellers. However, several striking relations were found with frequency of travel.

The SRRs for infectious diseases increased linearly with frequency of travel for both men (table 2) and women (table 3): SRRs 1.28, $1.54,1.97$ for men, and 1.16, 1.28, and 1.61 for women, respectively. A subset of theseintestinal diseases-followed a similar pattern; however, the trend increased monotonically and more steeply for women.

Claims for psychological disorders rose strongly with frequency of travel for both men and women. Among women, SRRs increased linearly with frequency of travel: SRRs 1.47 , 1.96, and 2.59. For men, psychological disorders among travellers completing one mission was twice that of non-travellers, and more than three times that of non-travellers for those completing two or more missions in 1993: SRRs 2.11, 3.13, and 3.06. A much smaller subset of diagnoses identified specifically as stress related (ICD-9 codes 308-309) also showed an increase with frequency of travel, although this was not monotonic among women.

Although disorders of the nervous system and sense organs seemed mildly correlated with frequency of travel for both men and women, the subset of diagnoses pertaining to the cornea, conjunctiva, and eyelid was moderately to strongly related to number of missions: SRRs for men, by mission frequency, were $1.73,2.42$, and 2.47 , and for women were 1.17 , 1.71 , and 2.01 , respectively.

Among men, SRRs also increased with frequency of travel for diseases of the respiratory system (SRRs 1.63, 1.82, and 2.05). When claims for diseases of the respiratory system among men were examined more closely, acute respiratory infections and other diseases of the upper respiratory tract (ICD-9 codes 460-478) increased with frequency of travel (SRRs 1.53, 1.79 , and 2.05).

Diseases of the skin and subcutaneous tissue increased with frequency of travel for both men (SRRs 1.48, 2.05, and 2.39) and women (SRRs 1.21, 1.23, and 1.34). For male travellers, claims for infections of the skin and subcutaneous tissue (ICD-9 codes 680-686) decreased with increasing frequency of travel (SRRs 3.32, 2.45, and 1.87), whereas claims 
for disorders of sweat and sebaceous glands (ICD codes 705 and 706) increased with frequency of travel (SRRs 1.23, 2.15, and 2.58).

The rate of claims for back injuries, specifically fractures, sprains, and strains (ICD codes $805-806,846-847$ ), was nearly $80 \%$ greater for male travellers than non-travellers and peaked for men who travelled on two to three missions (SRR 2.00).

\section{Discussion}

This study used diagnoses obtained from insurance claims to examine illness and diseases possibly associated with international travel. Claims rates for travellers compared with non-travellers were higher for all ICD-9 categories among men ( $80 \%$ overall), and for many categories among women ( $18 \%$ overall). Why travellers (and especially male travellers) file more medical insurance claims than their counterparts who remain at headquarters is not fully understood, and constitutes one of the major findings of this study.

Several explanations for the excess rates of claims found among travellers are plausible. Although a traveller with knowledge of a forthcoming business trip may visit a physician for a general physical examination, immunisation, health related travel advice, or renewal of a prescription, these types of visits would not result in increased rates of insurance claims filed across all ICD-9 categories. Travel may also exacerbate pre-existing or underlying medical conditions. Before departure travellers may be more inclined to seek medical attention rather than wait - as a non-traveller might - to see whether a condition worsens. After returning, travellers might seek medical attention for relatively minor problems out of concern that they may have contracted an illness while abroad. If this were the case, many of the excess claims should be for relatively minor conditions, or for assessment of an illness at an early stage of development; however, it was not possible to assess the severity of the conditions for which claims were filed. Furthermore, it was not possible to determine whether claims were filed by the travellers before, during, or after completion of their international missions.

Travellers may be more likely to use medical services than non-travellers because they have other different determinants of health from non-travellers. On the other hand, the excess rates of claims found among travellers may not be entirely due to greater use of medical services. Although specific categories of disease would be expected to be greater among travellers (travellers diarrhoea and other infectious diseases), many additional categories of illness may be indirectly related to travel due to the physical and mental stress of travel. Travellers often must endure dramatic and sudden changes in climate, daily activities, food and drink, and sleep patterns. Work requirements on the mission, possibly including communicating in a foreign language, operating in unfamiliar business and regional cultures, long hours in high intensity negotiations, and sepa- ration from the support of family and friends at home, may compound the physical and mental stress of travel.

To identify those diagnoses which might be related to some aspect of travel, we compared rates of claims by frequency of travel. Diseases with long latency periods as well as chronic conditions would not be expected to increase with increasing frequency of travel. For example, claims for cancers, although higher for travellers than non-travellers, decreased with increasing travel frequency. This is reasonable, as people with serious conditions may limit business travel.

The two categories for which the association with frequency of travel was strongest and most consistent for both men and women were infectious diseases and psychological disorders. Infectious diseases have long been associated with international travel. Travellers are more likely to be exposed to infectious organisms to which they are not immune, and the risk of contacting such organisms would logically increase with increased contact, either through more frequent missions or with longer periods abroad.

Given the stress of separation from family, language and cultural differences, and work demands abroad and on return, the excess rates of claims for psychological disorders are plausible. Sleep disorders, due to disruptions of the circadian system brought on by time zone changes and other stressors related to travel may contribute to psychological disturbances. ${ }^{10}$ One previous study has reported increased psychological disorders among French international travellers. ${ }^{11}$ More recently, Young ${ }^{12}$ showed psychiatric decompensation disorders in a population of travellers to Hawaii. East bound travellers were most likely to show symptoms of mania, and west bound travellers more likely to show symptoms of depression compared with comparable controls. In our study, we were unable to disaggregate travellers by destination or by direction of travel.

The threefold excess in claims due to psychological diagnoses among male travellers completing two or more missions is striking, as this group represents nearly half of all male staff at the World Bank. Women travelling on two or more missions generated twice the rate of psychological claims than non-travellers. For the subset of stress related disorders, the rate of claims increased with number of missions travelled for men. Although no such linear trend was found for female travellers, women who travelled the most filed nearly three times as many claims for stress related psychological disorders as non-travellers. Ongoing research on staff of the World Bank, including focus groups, suggests that the higher rates of psychological claims found among travellers may be related to $(a)$ a perceived excess workload, (b) multiple and competing demands on travellers' time, and (c) separation from family and friends. Travellers must reorder their personal and professional lives, frequently postponing or interrupting family, business, and social activities. These factors may contribute to stress. 
Karasek and Theorell ${ }^{14}$ proposed a model to measure job stress which incorporates psychological demands - that is, scope and pace of work-and decision latitude - that is, ability to control job tasks. Job stress is highest among workers with increased psychological demands and decreased decision latitude. Although employees of the World Bank who travel may experience a relatively high degree of autonomy with regard to specific job tasks, they may not be able to control when or where they travel and this may overburden their coping mechanisms. ${ }^{14} 15$

Other patterns of medical claims suggested an association with travel-such as diagnoses related to the cornea, conjunctiva, and eyelid. Factors such as sun exposure, low humidity environments aboard aircraft, or contamination of contact lenses with irritants or infectious agents may have a role. Skin diseases also seemed to be related to travel, especially among men. Skin diseases among travellers have been largely attributed to cutaneous larva migrans and pyodermas. $^{5}$ Others have noted sun damage and fungal infestations among international travellers, as well as other infectious and parasitic organisms. ${ }^{6}$ Our data suggested a strong travel related component for disorders of sweat and sebaceous glands, which may be associated with travel to hot climates.

Back injuries were increased among male travellers across all categories of mission frequency, but were not associated with travel among women except for those who travelled on at least four missions. Back sprains and strains may occur as the result of carrying luggage, and pre-existing back problems may be exacerbated by sitting for long hours on flights.

The findings from this cross sectional study cannot be interpreted as causal as the temporal relation between travel and diagnosis from the medical claim is unknown. Furthermore, the main finding that travellers file medical claims at a higher rate for nearly all categories of diagnoses must be better understood before results for more narrow categories of claims may be interpreted fully.
The correlation between increasing rates of claims and frequency of travel for infectious diseases and psychological disorders may be explained by travellers who seek medical attention before departure. More frequent travel, therefore, would lead to more claims filed. Because of the magnitude of the excess rates of claims, however, additional research is planned to identify the specific diagnostic categories most related to travel, as well as factors associated with physical illness and increased psychological stress. This research will evaluate risk factors such as number of time zones crossed and changes in climate, as well as determine the temporal relation between travel and the event for which the medical insurance claim is filed.

1 Wittner M, Tanowitz HB. Intestinal parasites in returned

2 travellers. Med Clin North Am 1992;76:1433-48. Hawkes S, Hart GJ, Bletsoe E, Shergold C, Johnson AM.
Risk behavior and STD acquisition in genitourinary clinic attenders who have travelled. Genitourin Med 1995;71:3514

3 Mulhall BP. Sexually transmissible diseases and travel. $\mathrm{Br}$ Med Bull 1993;49:395-411.

4 Cossar JH, Reid D, Fallon RJ, Bell EJ, Riding MH, Follett EAC, et al. A cumulative review of studies on travellers, their experience of illness and the implications of these findings. F Infect 1990;21:27-42.

5 Caumes E, Carrière J, Guermonprez G, Bricaire F, Danis $M$, Gentilini $M$. Dermatoses associated with travel to tropical countries: a prospective study of the diagnosis and management of 269 patients presenting to a tropical management of 269 patients presenting

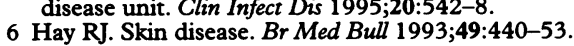

7 Lockwood DNJ, Keystone JS. Skin problems in returning Lockwood DNJ, Keystone JS. Skin problems in
travelers. Med Clin North Am 1992;76:1393-411.

8 Black J. Deep-vein thrombosis and pulmonary embolism. Lancet 1993;342:352-3.

9 Cruickshank JM, Gorlin R, Jennett B. Air travel and thrombotic episodes: the economy class syndrome. Lancet 1988; ii:497-8.

10 Naitoh P, Kelly TL, Englund C. Health effects of sleep deprivation. Occup Med 1990;5:209-37.

11 Sauteraud A, Hajjar M. Troubles psychotique: fréquence plus élevée lors des voyages en Asie. Presse Méd 1992;21: 805-10.

12 Young DM. Psychiatric morbidity in travelers to Honolulu, Hawaii. Compr Psychiatry 1995;36:224-8.

13 Bewes PC. Trauma and accidents: practical aspects of the prevention and management of trauma associated with travel. Br Med Bull 1993;49;454-64.

14 Karasek R, Theorell T. Healthy work: stress, productivity and the reconstruction of working life. New York: Basic Books, 1990.

15 Evanoff BA, Rosenstock L. Psychophysiologic stressors and work organization. In: Rosenstock L, Cullen MR, eds. Textbook of clinical occupational and environmental medicine. Philadelphia: WB Saunders, 1994:717-28. 